\title{
PRESSÃO ARTERIAL SISTÓLICA NOTURNA E DOENÇA CEREBROVASCULAR SUBCLÍNICA: O ESTUDO DE ANORMALIDADES CARDIOVASCULARES E LESÕES CEREBRAIS
}

\author{
NIGHT-TIME SYSTOLIC BLOOD PRESSURE AND SUBCLINICAL \\ CEREBROVASCULAR DISEASE: THE CARDIOVASCULAR ABNORMALITIES AND \\ BRAIN LESIONS (CABL) STUDY
}

Nakanishi K, Jin Z, Homma S, Elkind MSV, Rundek T, Schwartz JE, et al. Eur Heart J Cardiouasc Imaging. 2019 Jul 1;2O(7):765-771. doi: 10.1093/ehjci/jey221

José Fernando Vilela Martin

\section{RESUMO}

Adaptado do original - tradução livre

Introdução: Embora a pressão arterial (PA) ambulatorial seja um melhor preditor de desfechos cardiovasculares do que a pressão arterial de consultório, sua associação com a doença cerebrovascular subclínica não está esclarecida. Assim, esse estudo investigou a associação dos valores da PA de consultório e ambulatorial com a doença cerebrovascular subclínica em uma coorte populacional predominantemente idosa, sem histórico de acidente vascular cerebral prévio. Material e Métodos: 828 participantes foram submetidos à monitorização ambulatorial da PA por 24 horas (MAPA), ecocardiograma, ressonância magnética cerebral no estudo Cardiac Abnormalities and Brain Lesion (CABL). PA de vigília, PA durante o sono e de 24 horas, padrão de descenso noturno, elevação matutina (EM) e variabilidade da PA de 24 horas foram avaliados. A doença cerebrovascular subclínica foi definida como infarto cerebral silencioso (ICS) e volume de hiperintensidade da substância branca (VHSB). A associação das medidas da PA com a presença de ICS e o quartil superior do log-VHSB (log-VHSB 4) também foi analisada. Resultados: ICSs foram detectados em 111 pacientes (13,4\%). Na análise multivariável, apenas a PA sistólica (PAS) noturna esteve significantemente associada ao ICS [odds ratio (OR): 1,15 para cada 10mmHg, $\mathrm{P}=0,042$ ], independente de fatores de risco cardiovascular e parâmetros ecocardiográficos avaliados. Embora PA na vigília, durante o sono, PA de 24 horas e o padrão não dipper tenham sido significantemente associados ao log-VHSB 4, a PAS noturna apresentou a associação mais forte (OR: 1,21 para cada $10 \mathrm{mmHg}, \mathrm{P}=0,003)$ e foi o único preditor independente comparado aos outros parâmetros da PA. Medidas da PA de consultório, EM e variabilidade da PA não foram associadas à doença cerebrovascular subclínica nas análises ajustadas. Conclusão: A PAS elevada durante o sono apresenta forte associação com a doença cerebrovascular subclínica. O estudo conclui que a PAS noturna avaliada pela MAPA permite identificar indivíduos com maior risco de lesão cerebral hipertensiva.

\section{ANÁLISE CRÍTICA}

Esse estudo demonstrou que a PAS noturna foi um preditor mais forte da presença de doença cerebrovascular subclínica em uma amostra predominantemente idosa sem história de acidente vascular cerebral (AVC). A associação entre PAS durante o sono e doença cerebral subclínica ocorreu independente de outros fatores de risco cardiovascular tradicionais e de alterações ecocardiográficas relacionadas à hipertensão. ${ }^{1}$

Já se conhece que a PA obtida pela MAPA é melhor preditor de doenças cardiovasculares e de mortalidade do que a PA de consultório. ${ }^{2,3}$ O primeiro relato desse achado data de 1988, quando O'Brien e colaboradores mostraram que pacientes hipertensos com menor queda noturna da PA cursavam com maior prevalência de AVC e os denominou de "não dippers". ${ }^{4}$ Desde então, vários estudos investigaram a associação entre a falta do descenso noturno (DN) e doenças cerebrovasculares. No entanto, os estudos sobre a presença de não dipper e risco de AVC tem apresentado resultados conflitantes. ${ }^{5-7}$

1. Faculdade de Medicina de São José do Rio Preto (FAMERP), São Paulo, SP, Brasil.

Correspondência: Faculdade de Medicina de São José do Rio Preto (FAMERP), São Paulo, SP Brasil. Av. Brg. Faria Lima, 5416 - Vila Sao Pedro, São José do Rio Preto - SP, 15090-OOO. vilelamartin@uol.com.br

http://dx.doi.org/10.47870/1519-7522/2020270276-7 
Em um estudo, a razão PAS sono/vigília não foi associada ao AVC em 7.458 indivíduos durante o acompanhamento médio de 9,6 anos. ${ }^{5}$ Outro estudo mostrou que a razão PAS sono/ vigília foi preditor da ocorrência de AVC em uma metanálise de 17.312 hipertensos. ${ }^{6}$ Nosso grupo avaliou a prevalência de DN em 163 indivíduos hipertensos e mostrou que a ausência de DN associou-se de maneira independente às lesões em órgãos-alvo analisadas - AVC e infarto do miocárdio. Embora a PAS no sono tenha sido maior no grupo não dipper, ela não se associou aos eventos avaliados. ${ }^{7}$

Entretanto, há evidências crescentes de que o nível médio da PA durante o sono, em vez do padrão noturno de não dipper, pode ser o maior preditor do risco de AVC em indivíduos com ou sem hipertensão. Fagard e colaboradores demonstraram que uma diferença de $17,6 \mathrm{mmHg}$ na PAS noturna foi associada a um aumento do risco de AVC em 3.468 hipertensos, enquanto a razão PAS sono/vigília não foi associada a AVC após o ajuste para PAS de 24 horas. ${ }^{8}$

No presente estudo, os infartos cerebrais silenciosos (ICSs) e os volumes de hiperintensidade da substância branca (VHSBs), definidos por ressonância magnética cerebral, estão comumente presentes em adultos idosos e são importantes marcadores da doença cerebral de pequenos vasos. Embora os ICSs e VHSBs não estejam tipicamente ligados a sintomas evidentes de AVC clínico, eles não são totalmente silenciosos ou benignos, pois geralmente estão associados a sintomas neurológicos sutis e maior risco de AVC, comprometimento cognitivo, demência e morte. ${ }^{9-11}$

Vários mecanismos podem explicar a razão da PA noturna elevada se associar à doença cerebrovascular subclínica. Hiperatividade simpática persistente pode refletir melhor o estresse mecânico sobre a parede arterial do que a PA de vigília. ${ }^{12}$

\section{REFERÊNCIAS}

1. Nakanishi K, Jin Z, Homma S, Elkind MSV, Rundek T, Schwartz JE, et al. Night-time systolic blood pressure and subclinical cerebrovascular disease: the Cardiovascular Abnormalities and Brain Lesions (CABL) study. Eur Heart J Cardiovasc Imaging. 2019;20(7):765-71.

2. O'Brien E, Parati G, Stergiou G, Asmar R, Beilin L, Bilo G, et al. European Society of Hypertension position paper on ambulatory blood pressure monitoring. J Hypertens 2013;31:1731-68.

3. Shimbo D, Abdalla M, Falzon L, Townsend RR, Muntner P. Role of ambulatory and home blood pressure monitoring in clinical practice: a narrative review. Ann Intern Med 2015;163:691-700.

4. O'Brien E, Sheridan J, O'Malley K. Dippers and non-dippers. Lancet $1988 ; 2: 397$

5. Boggia J, Li Y, Thijs L, Hansen TW, Kikuya M, Bjorklund-Bodegard K, et al. Prognostic accuracy of day versus night ambulatory blood pressure: a cohort study. Lancet 2007;370:1219-29.

6. Salles GF, Reboldi G, Fagard RH, Cardoso CR, Pierdomenico SD, Verdecchia $\mathrm{P}$, et al. Prognostic effect of the nocturnal blood pressure fall in hypertensive patients: the ambulatory blood pressure collaboration in patients with hypertension (ABC-H) meta-analysis. Hypertension 2016;67:693-700.

7. Vaz-de-Melo RO, Toledo JC, Loureiro AA, Cipullo JP, Moreno Júnior $\mathrm{H}$, Martin JF. Absence of Nocturnal Dipping is Associated with Stroke and Myocardium Infarction. Arq Bras Cardiol 2010; 94: 79-85.

8. Fagard RH, Celis H, Thijs L, Staessen JA, Clement DL, De Buyzere ML, et al. Daytime and nighttime blood pressure as predictors of death and causespecific cardiovascular events in hypertension. Hypertension 2008;51:55-61.

9. Vermeer SE, Hollander M, van Dijk EJ, Hofman A, Koudstaal PJ, Breteler MMB, et al. Silent brain infarcts and white matter lesions increase stroke risk in the general population: the Rotterdam Scan Study. Stroke 2003;34:1126-9.
Outros fatores podem estar presentes, entre eles, função endotelial prejudicada, ativação plaquetária e resposta inflamatória aumentada, e maior sensibilidade ao sal. ${ }^{13-15}$ Além disso, distúrbio do sono também pode estar envolvido na associação observada. ${ }^{16}$

Os autores também discutem sobre a elevação matutina (EM) da PA, mostrando que apesar de ser considerada um fenômeno fisiológico após o despertar, uma elevação acentuada e rápida pode se associar ao aumento do risco de doença cerebrovascular. Trabalhos têm demonstrado que EM elevada é preditor da incidência de AVC em pacientes hipertensos tratados $^{17}$ e nos indivíduos com $E M \geq 55 \mathrm{mmHg}$ comparados àqueles sem EM, houve maior incidência de múltiplos ICSs. ${ }^{18}$ No presente estudo, a EM não foi significativamente associada à doença cerebral subclínica. Os autores justificam essa discrepância pelas diferentes populações e comorbidades avaliadas, uma vez que os asiáticos parecem ter maior PA matinal comparado com ocidentais.

A variabilidade ambulatorial da PA também foi avaliada no presente estudo e não demonstrou associação independente com doença cerebrovascular subclínica, sugerindo que valores médios elevados da PA, principalmente à noite, podem ser determinantes mais importantes da doença cerebral silenciosa do que flutuações da PA nas $24 \mathrm{~h}$.

Os autores também discutem a importância de acompanhamento mais próximo de indivíduos com PAS noturna elevada, uma vez que ICSs e VHSBs carregam um risco aumentado de AVC. Assim, terapia anti-hipertensiva com foco no período noturno para melhor controle da PA durante o sono, controle mais intensivo dos fatores de risco e tratamento para apneia do sono podem ser condutas úteis para reduzir a PAS noturna. A adoção dessas medidas pode exercer efeitos benéficos sobre a doença cerebrovascular subclínica ao longo do tempo.

10. Vermeer SE, Prins ND, den Heijer T, Hofman A, Koudstaal PJ, Breteler MM. Silent brain infarcts and the risk of dementia and cognitive decline. N Engl J Med 2003;348:1215-22.

11. Wright CB, Dong C, Perez EJ, De Rosa J, Yoshita M, Rundek T, et al. Subclinical cerebrovascular disease increases the risk of incident stroke and mortality: the Northern Manhattan study. J Am Heart Assoc 2017;6:e004069.

12. Dodt C, Breckling U, Derad I, Fehm HL, Born J. Plasma epinephrine and norepinephrine concentrations of healthy humans associated with nighttime sleep and morning arousal. Hypertension 1997;30:71-6.

13. Maio R, Perticone M, Sciacqua A, Tassone EJ, Naccarato P, Bagnato C, et al. Oxidative stress impairs endothelial function in nondipper hypertensive patients. Cardiovasc Ther 2012;30:85-92.

14. Kaya MG, Yarlioglues M, Gunebakmaz O, Gunturk E, Inanc T, Dogan A, et al. Platelet activation and inflammatory response in patients with non-dipper hyper- tension. Atherosclerosis 2010;209:278-82.

15. Sachdeva A, Weder AB. Nocturnal sodium excretion, blood pressure dipping, and sodium sensitivity. Hypertension 2006;48:527-33.

16. Portaluppi F, Provini F, Cortelli P, Plazzi G, Bertozzi N, Manfredini R, et al. Undiagnosed sleep-disordered breathing among male nondippers with essential hypertension. J Hypertens. 1997;15:1227-33.

17. Pierdomenico SD, Pierdomenico AM, Cuccurullo F. Morning blood pressure surge, dipping, and risk of ischemic stroke in elderly patients treated for hypertension. Am J Hypertens. 2014;27:564-70.

18. Kario K, Pickering TG, Umeda Y, Hoshide S, Hoshide $Y$, Morinari M, et al. Morning surge in blood pressure as a predictor of silent and clinical cerebrovascular disease in elderly hypertensives: a prospective study. Circulation. 2003;107:1401-6. 